\title{
Fractional Ecosystem Model and Its Solution by Homotopy Perturbation Method
}

\author{
Pius h Kumar Singh*, T Som \\ Department of Applied Mathematics, Indian Institute of Technology (Banaras Hindu University), Varanasi, 221005, India
}

\begin{abstract}
In the present paper we propose an algorithm based on Homotopy Perturbation Method to solve the fractional Ecosystem model and show the efficiency and accuracy of the algorithm. This Ecosystem model is solved with time fractional derivatives in the sense of Caputo. The nonlinear terms can be easily handled by the using He's Polynomials. The numerical solutions of the Ecosystem model reveal that only a few iterations are suffic ient to obtained accurate approximate analytical solutions. The numerical results obtained are presented graphically. The four different cases are considered and proved that the method is extremely effective due to fractional approach and performance. Comparing the methodology (FHPM) with the some known technique (HPM) shows that the present approach is effective and powerful. The proposed scheme finds the solution with the help of Mathematica and without any restrictive assumptions. We implement it to four different problems.
\end{abstract}

Keywords Nonlinear System, Fractional Ecosystem Model, Homotopy Perturbation Method, Fractional Integral Operator

\section{Introduction}

Ecosystem models play an important role to know the dynamics of the system. Such models are formed by combining known ecological relations with data gathered from field observations ([1]-[8]). These models are then used to make predictions about the dynamics of the real system. However, the study of deficiencies in the model leads to the generation of hypotheses about the possible ecological relations that are not yet known or well understood. Models enable researchers to simulate the conclusions of large-scale experiments that might be too expensive to perform on a real ecosystem. These also enable the simulation of ecological processes over very long period of time (may be some process may take centuries of time in reality), However this can be done in a matter of minutes in a computer model. Ecosystem models have applications in a wide variety of disciplines, in particular, to natural resource management. An important aspect any model evaluation is the validation, that is, the process of confirming that the model behaviour corresponds to reality. The results of the model can be validated by comparison with field data, but this technique limits the validation to a particu lar set of field conditions. We obtain a sequence of equations in which the solution at any stage is close to the solution at nearby stages. In the present

* Corresponding author:

piushsingh82@gmail.com (Piush Kumar Singh)

Published online at http://journal.sapub.org/ije

Copyright (C) 2012 Scientific \& Academic Publishing. All Rights Reserved paper we discuss the fractional Ecosystem model and solve it using Homotopy Perturbation Method.

We consider the following fractional ecosystem model given by fractional initial value problem of the type:

$$
D^{\alpha} x(t)=f(x(t), y(t)) \quad D^{\alpha} y(t)=g(x(t), y(t))
$$
with initial conditions $x(0)=1, y(0)=-1$. Here $\mathrm{f}$ and $\mathrm{g}$ are arbitrary functions, $D^{\alpha}$ denotes the fractional derivative in the sense of Caputo.

\section{Basic Definitions}

In this section, we give the related definitions and properties of the fractional calculus from [9].

Definition 2.1. A real function $h(t), t>0$ is said to be in the space $C_{\mu}, \mu \in R$, if there exists a real number $p>\mu, \quad$ such that $h(t)=t^{p} h_{1}(t), \quad$ where $h_{1}(t) \in C(0, \infty)$, and it is said to be in the space $C_{\mu}^{n}$ if and only if $h^{(n)} \in C_{\mu}, n \in N$.

Definition 2.2 The Riemann-Liouville fractional integral operator $\left(J^{\alpha}\right) \quad$ of order $\alpha \geq 0$, of a function, $h \in C_{\mu}, \mu \geq-1$, is defined as

$$
J^{\alpha} h(t)=\frac{1}{\sqrt{(\alpha)}} \int_{0}^{t}(t-\tau)^{\alpha-1} h(\tau) d \tau \quad(\alpha>0),
$$


$J^{0} h(t)=h(t)$, where $\sqrt{(z)}$ is the well-known Gamma function.

Some of the properties of the operator $J^{\alpha}$, which we need here, are as follows:

For $h \in C_{\mu}, \mu \geq-1, \alpha, \beta \geq 0$ and $\gamma \geq-1$ :

(1) $J^{\alpha} J^{\beta} h(t)=J^{\alpha+\beta} h(t)$,

(2) $J^{\alpha} J^{\beta} h(t)=J^{\beta} J^{\alpha} h(t)$,

(3) $J^{\alpha} t^{\gamma}=(\sqrt{(\gamma+1)} / \sqrt{(\alpha+\gamma+1)}) t^{\alpha+\gamma}$.

Definition 2.3. [10] The fractional derivative $D^{\alpha}$ of $h(t)$ in the Caputo's sense is defined as

$D^{\alpha} h(t)=\frac{1}{\sqrt{(n-\alpha)}} \int_{0}^{t}(t-\tau)^{n-\alpha-1} h^{(n)}(\tau) d \tau, \quad$ for $n-1<\alpha \leq n, n \in N, t>0, h \in C_{-1}^{n}$.

The following are the two basic properties of the Caputo's fractional derivative [10]:

(1) Let $h \in C_{-1}^{n}, n \in N$. Then $D^{\alpha} h, 0 \leq \alpha \leq n$ is well defined and $D^{\alpha} h \in C_{-1}$.

(2) Let $n-1<\alpha \leq n, n \in N$ and $h \in C_{\mu}^{n}, \mu \geq-1$. This leads to

$$
\left(J^{\alpha} D^{\alpha}\right) h(t)=h(t)-\sum_{k=0}^{n-1} h^{(k)}\left(0^{+}\right) \frac{t^{k}}{k !} .
$$

\section{Analysis of HPM}

The Homotopy Perturbation Method (HPM), which provides analytically an approximate solution, is applied to various nonlinear problems ([11]-[14]). In this section, we introduce a reliable algorithm to handle the nonlinear ecosystems in a realistic and efficient way. The proposed algorith $\mathrm{m}$ will then be used to investigate the system given by:

$$
\begin{aligned}
& D^{\alpha} y_{1}(t)=\sum_{i=1}^{n} a_{1 i}(t) y_{i}+g_{1}\left(t, y_{1}, y_{2} \ldots y_{n}\right) \\
& D^{\alpha} y_{2}(t)=\sum_{i=1}^{n} a_{2 i}(t) y_{i}+g_{2}\left(t, y_{1}, y_{2} \ldots y_{n}\right) \\
& D^{\alpha} y_{3}(t)=\sum_{i=1}^{n} a_{3 i}(t) y_{i}+g_{3}\left(t, y_{1}, y_{2} \ldots y_{n}\right) \\
& D^{\alpha} y_{n}(t)=\sum_{i=1}^{n} a_{n i}(t) y_{i}+g_{n}\left(t, y_{1}, y_{2} \ldots y_{n}\right)
\end{aligned}
$$

subject to the initial condition,

$$
y_{1}(0)=c_{1}, \quad y_{2}(0)=c_{2}, \quad y_{n}(0)=c_{n},
$$

where $g_{i}$ is a nonlinear function for each $i=1,2, \ldots n$. Using the homotopy perturbation method we can construct the following ho motopy for $i=1,2, \ldots n$,

$$
D^{\alpha} y_{i}(t)-\sum_{j=1}^{n} a_{i j}(t) y_{j}=p g_{i}\left(t, y_{1,} y_{2} \ldots y_{n}\right),
$$

where $p \in[0,1]$ is the homotopy parameter, which takes values from zero to unity. In case $p=0$, equation (3) becomes a linear equation given by:

$$
D^{\alpha} y_{i}(t)=\sum_{j=1}^{n} a_{i j}(t) y_{j},
$$

and when $p$ is 1 , equation (3) turns out to be the original equation given as system (1). Assuming that the solution of the system (1) is a power series in $p$ given by:

$$
y_{i}=y_{i}^{(0)}+p y_{i}^{(1)}+p^{2} y_{i}^{(2)}+p^{3} y_{i}^{(3)}+p^{4} y_{i}^{(4)}+\ldots
$$

Substituting equation (5) in equation (3), and equating the terms having identical powers of $p$, we obtain a series of linear equations in the form:

$$
\begin{gathered}
p^{0}: D^{\alpha} y_{i}^{(0)}(t)=\sum_{j=1}^{n} a_{i j}(t) y_{j}{ }^{(0)}, y_{i}{ }^{(0)}(0)=c_{i}, \\
p^{1}: D^{\alpha} y_{i}^{(1)}(t) \\
=\sum_{j=1}^{n} a_{i j}(t) y_{j}^{(1)}+g_{i}^{(1)}\left(t, y^{(0)}\right), y_{i}^{(1)}(0)=0, \\
p^{2}: D^{\alpha} y_{i}^{(2)}(t) \\
=\sum_{j=1}^{n} a_{i j}(t) y_{j}^{(2)}+g_{i}^{(2)}\left(t, y^{(0)}, y^{(1)}\right), y_{i}^{(2)}(0)=0 \\
p^{3}: D^{\alpha} y_{i}^{(3)}(t) \\
=\sum_{j=1}^{n} a_{i j}(t) y_{j}^{(3)}+g_{i}^{(3)}\left(t, y^{(0)}, y^{(1)}, y^{(2)}\right), y_{i}^{(3)}(0)=0
\end{gathered}
$$

where the function $g_{i}^{(1)}, g_{i}^{(2)}, g_{i}^{(3)} \ldots$ satisfy the following equation:

$$
\begin{aligned}
& g_{i}\left(\begin{array}{l}
t, y_{1}{ }^{(0)}+p y_{1}^{(1)}+p^{2} y_{1}^{(2)}+p^{3} y_{1}^{(3)}+\ldots, \\
y_{n}{ }^{(0)}+p y_{n}{ }^{(1)}+p^{2} y_{n}{ }^{(2)}+\ldots
\end{array}\right) \\
& =g_{i}{ }^{1}\left(t, y_{1}{ }^{(0)}, y_{2}{ }^{(0)}, \ldots, y_{n}{ }^{(0)}\right) \\
& +p g_{i}^{(2)}\left(t, y_{1}{ }^{(0)}, y_{2}{ }^{(0)}, \ldots, y_{n}{ }^{(0)}, t, y_{1}{ }^{(1)}, y_{2}{ }^{(1)}, \ldots, y_{n}{ }^{(1)}\right) \\
& =+p^{2} g_{i}^{(3)}\left(\begin{array}{l}
t, y_{1}^{(0)}, y_{2}^{(0)}, \ldots, y_{n}^{(0)}, y_{1}^{(1)}, \\
y_{2}^{(1)}, \ldots, y_{n}^{(1)}, y_{1}^{(2)}, y_{2}^{(2)}, \ldots, y_{n}^{(2)}
\end{array}\right)+\ldots
\end{aligned}
$$

Now taking $p=1$ in the equation (5) it gives the solution of the system (1). It is obvious that the above linear equations are easy to solve, and the components $y_{i}^{(k)}$, 
$k \geq 0$, of the homotopy perturbation solution can be completely determined as a series solution,

Finally, we approximate the solution $y_{i}(t)=\sum_{k=0}^{\infty} p^{k} y_{i}^{(k)}(t)$ by the truncated series

$$
\phi_{i}(t)=\sum_{k=0}^{N-1} p^{k} y_{i}^{(k)}(t)
$$

It is also useful, for the system (1) to construct the homotopy, for $i=1,2, \ldots, n$

$$
D^{\alpha} y_{i}(t)-p \sum_{j=1}^{n} a_{i j}(t) y_{j}=p g_{i}\left(t, y_{1,} y_{2} \ldots y_{n}\right)
$$

where $p \in[0,1]$. In this case, the term $\sum_{j=1}^{n} a_{i j}(t) y_{j}^{(0)}$ is combined with the component $y_{i}^{(1)}$ and the term $\sum_{j=1}^{n} a_{i j}(t) y_{j}^{(1)}$ is combined with the component $y_{i}^{(2)}$ and so on. This variation reduces the number of terms in each component and also minimizes the size of calculations. By substituting equation (5) in equation (7) we obtain the following series of linear equations:

$$
\begin{gathered}
p^{0}: D^{\alpha} y_{i}^{(0)}(t)=0, y_{i}^{(0)}(0)=c_{i}, \\
p^{1}: D^{\alpha} y_{i}^{(1)}(t)= \\
\sum_{j=1}^{n} a_{i j}(t) y_{j}^{(0)}+g_{i}^{(1)}\left(t, y^{(0)}\right), y_{i}^{(1)}(0)=0 \\
p^{2}: D^{\alpha} y_{i}^{(2)}(t)= \\
\sum_{j=1}^{n} a_{i j}(t) y_{j}^{(1)}+g_{i}^{(2)}\left(t, y^{(0)}, y^{(1)}\right), y_{i}^{(2)}(0)=0 \\
p^{3}: D^{\alpha} y_{i}^{(3)}(t)= \\
\sum_{j=1}^{n} a_{i j}(t) y_{j}^{(2)}+g_{i}^{(3)}\left(t, y^{(0)}, y^{(1)}, y^{(2)}\right), y_{i}^{(3)}(0)=0
\end{gathered}
$$

$p^{4}: D^{\alpha} y_{i}^{(4)}(t)=$

$\sum_{j=1}^{n} a_{i j}(t) y_{j}^{(3)}+g_{i}^{(4)}\left(t, y^{(0)}, y^{(1)}, y^{(2)}, y^{(3)}\right), \quad y_{i}^{(4)}(0)=0$

\section{Numerical Example}

In this section we show the application of the algorithm based on HPM given above to solve four different linear and non linear fractional Ecosystem model.

Example 4.1. Consider the linear system [15]

$$
\begin{aligned}
& D^{\alpha} x(t)=y(t), \\
& \qquad D^{\alpha} y(t)=2 x(t)-y(t),
\end{aligned}
$$

subject to the initial conditions

$$
x(0)=1, y(0)=-1,
$$

We construct the Homotopy

$$
\begin{aligned}
& D^{\alpha} x(t)=p y(t) \\
& D^{\alpha} y(t)=p(2 x(t)-y(t))
\end{aligned}
$$

To solve the system given in Eq. (8) we construct the Ho motopy as given in Eq. (7) putting Eq. (5) then initial condition (9) in Eq. (7) and then comparing the like powers of $p$ we get,

$$
\begin{gathered}
p^{0}: D^{\alpha} x^{(0)}=0, p^{0}: D^{\alpha} y^{(0)}=0 \\
p^{1}: D^{\alpha} x^{(1)}=y^{(0)}, p^{1}: D^{\alpha} y^{(1)}=2 x^{(0)}-y^{(0)} \\
p^{2}: D^{\alpha} x^{(2)}=y^{(1)}, p^{2}: D^{\alpha} y^{(2)}=2 x^{(1)}-y^{(1)} \\
p^{3}: D^{\alpha} x^{(3)}=y^{(2)}, p^{3}: D^{\alpha} y^{(3)}=2 x^{(2)}-y^{(2)} \\
p^{4}: D^{\alpha} x^{(4)}=y^{(3)}, p^{4}: D^{\alpha} y^{(4)}=2 x^{(3)}-y^{(3)} \\
p^{5}: D^{\alpha} x^{(5)}=y^{(4)}, p^{5}: D^{\alpha} y^{(5)}=2 x^{(4)}-y^{(4)}
\end{gathered}
$$

Using the operator $J_{t}^{\alpha}$, the inverse operator of $D^{\alpha}$, on both side of linear equations from $(10)-(15)$ with initial conditions (9) we get

$$
\begin{gathered}
x^{(0)}=x^{(0)}(0)=1, \quad x^{(1)}=J_{t}^{\alpha}\left[y^{(0)}\right]=(-1) \frac{t^{\alpha}}{\overline{(1+\alpha)}}, \\
x^{(2)}=J_{t}^{\alpha}\left[y^{(1)}\right]=(3) \frac{t^{2 \alpha}}{\sqrt{(1+2 \alpha)}}, x^{(3)}=J_{t}^{\alpha}\left[y^{(2)}\right]=(-5) \frac{t^{3 \alpha}}{\sqrt{(1+3 \alpha)}}, \\
x^{(4)}=J_{t}^{\alpha}\left[y^{(3)}\right]=(11) \frac{t^{4 \alpha}}{\sqrt{(1+4 \alpha)}}, \quad x^{(5)}=J_{t}^{\alpha}\left[y^{(4)}\right]=(-21) \frac{t^{5 \alpha}}{\sqrt{(1+5 \alpha)}},
\end{gathered}
$$




$$
\begin{gathered}
y^{(0)}=y^{(0)}(0)=-1, \quad y^{(1)}=J_{t}^{\alpha}\left[2 x^{(0)}-y^{(0)}\right]=(3) \frac{t^{\alpha}}{\sqrt{(1+\alpha)}}, \\
y^{(2)}=J_{t}^{\alpha}\left[2 x^{(1)}-y^{(1)}\right]=(-5) \frac{t^{2 \alpha}}{\sqrt{(1+2 \alpha)}}, y^{(3)}=J_{t}^{\alpha}\left[2 x^{(2)}-y^{(2)}\right]=(11) \frac{t^{3 \alpha}}{\sqrt{(1+3 \alpha)}}, \\
y^{(4)}=J_{t}^{\alpha}\left[2 x^{(3)}-y^{(3)}\right]=(-21) \frac{t^{4 \alpha}}{\sqrt{(1+4 \alpha)}}, y^{(5)}=J_{t}^{\alpha}\left[2 x^{(4)}-y^{(4)}\right]=(43) \frac{t^{5 \alpha}}{\sqrt{(1+5 \alpha)}},
\end{gathered}
$$

The solution of $(8)$ is given by:

$$
\begin{gathered}
x(t)=\sum_{i=0}^{\infty} x^{(i)}(t)=1-\frac{t^{\alpha}}{\sqrt{(1+\alpha)}}+3 \frac{t^{2 \alpha}}{\sqrt{(1+2 \alpha)}}-5 \frac{t^{3 \alpha}}{\sqrt{(1+3 \alpha)}}+11 \frac{t^{4 \alpha}}{\sqrt{(1+4 \alpha)}}-21 \frac{t^{5 \alpha}}{\sqrt{(1+5 \alpha)}}+\ldots \\
y(t)=\sum_{i=0}^{\infty} y^{(i)}(t) \quad=-1+3 \frac{t^{\alpha}}{\sqrt{(1+\alpha)}}-5 \frac{t^{2 \alpha}}{\sqrt{(1+2 \alpha)}}+11 \frac{t^{3 \alpha}}{\sqrt{(1+3 \alpha)}}-21 \frac{t^{4 \alpha}}{\sqrt{(1+4 \alpha)}}+43 \frac{t^{5 \alpha}}{\sqrt{(1+5 \alpha)}}+\ldots
\end{gathered}
$$

the graph of which is given below:

From Fig. 1 and Fig. 2 we see that the solution obtained by the proposed algorithm for $\alpha=1$ is same as the solution obtained by [16]. Also figures 1 and 2 show the solution for other different values of $\alpha$. Fig 1 and 2 show the solutions in red by HPM of the original system and by HPM of the fractional ecosystem model by blue thick dots.

Example 4.2. Consider the predator - prey system [15].

$$
D^{\alpha} x(t)=x(t)-x(t) y(t) \text {, }
$$

$$
D^{\alpha} y(t)=-y(t)+x(t) y(t)
$$

subject with the initial conditions

$$
x(0)=1, \quad y(0)=0.5,
$$

We construct the Homotopy

$$
\begin{aligned}
& D^{\alpha} x(t)=p(x(t)-x(t) y(t)), \\
& D^{\alpha} y=p(-y(t)+x(t) y(t)),
\end{aligned}
$$

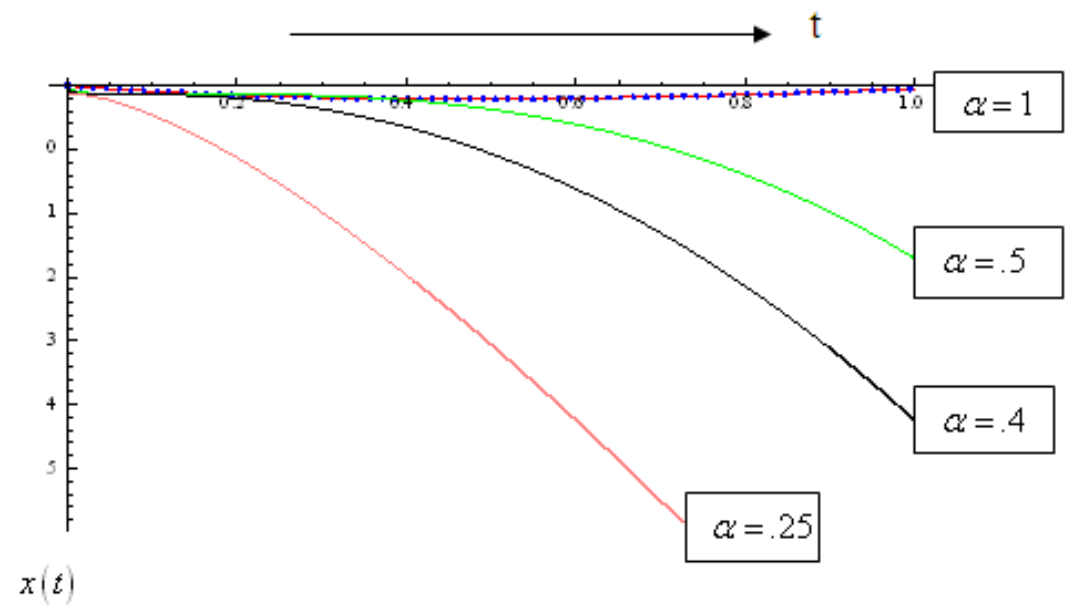

Figure 1. Plot of $x(t)$ vs $t$ 


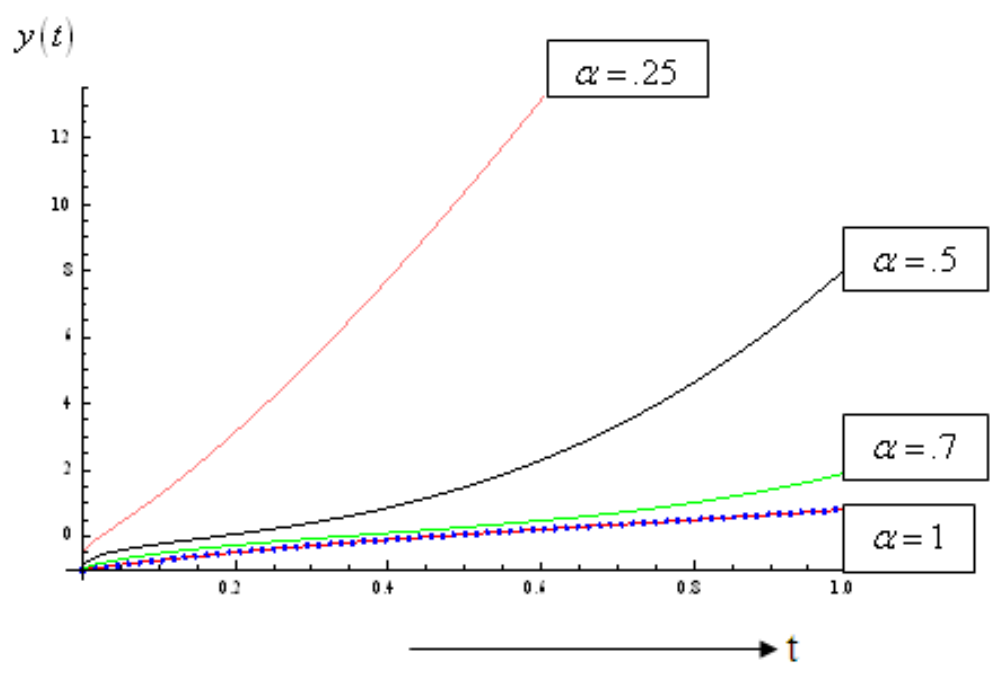

Figure 2. Plot of $y(t)$ vs $t$

To solve the system given in Eq. (16) we construct the Homotopy as given in Eq. (7) putting Eq. (5) then intial condition (17) in Eq. (7) and then comparing the like powers of $p$ we get,

$$
\begin{aligned}
& p^{0}: D^{\alpha} x^{(0)}=0, p^{0}: D^{\alpha} y^{(0)}=0, \\
& p^{1}: D^{\alpha} x^{(1)}=x^{(0)}-x^{(0)} y^{(0)}, \\
& p^{1}: D^{\alpha} y^{(1)}=x^{(0)} y^{(0)}-y^{(0)} \\
& p^{2}: D^{\alpha} x^{(2)}=x^{(1)}-x^{(0)} y^{(1)}-x^{(1)} y^{(0)}, \\
& p^{2}: D^{\alpha} y^{(2)}=y^{(0)} x^{(1)}-y^{(1)}+x^{(0)} y^{(1)} \\
& p^{3}: D^{\alpha} x^{(3)}=x^{(2)}-x^{(0)} y^{(2)}-x^{(1)} y^{(1)}-x^{(2)} y^{(0)}, \\
& p^{3}: D^{\alpha} y^{(3)}=y^{(0)} x^{(2)}-y^{(2)}+x^{(0)} y^{(2)}-x^{(1)} y^{(1)},
\end{aligned}
$$

Now using the operator $J_{t}^{\alpha}$, the inverse operator of $D^{\alpha}$, on both side of linear equations from $(18)-(21)$ with in itial condition (17) we get,

$$
\begin{gathered}
x^{(0)}=x^{(0)}(0)=1, x^{(1)}=J_{t}^{\alpha}\left[x^{(0)}-x^{(0)} y^{(0)}\right]=(0.5) \frac{t^{\alpha}}{\sqrt{(1+\alpha)}}, \\
x^{(2)}=J_{t}^{\alpha}\left[x^{(1)}-x^{(0)} y^{(1)}-x^{(1)} y^{(0)}\right]=(0.25) \frac{t^{2 \alpha}}{\sqrt{(1+2 \alpha)}}, \\
x^{(3)}=J_{t}^{\alpha}\left[x^{(2)}-x^{(0)} y^{(2)}-x^{(1)} y^{(1)}-x^{(2)} y^{(0)}\right]=(0.125) \frac{t^{3 \alpha}}{\sqrt{(1+3 \alpha)}}, \\
y^{(0)}=y^{(0)}(0)=0.5, y^{(1)}=J_{t}^{\alpha}\left[x^{(0)} y^{(0)}-y^{(0)}\right]=0 \\
y^{(2)}=J_{t}^{\alpha}\left[y^{(0)} x^{(1)}-y^{(1)}+x^{(0)} y^{(1)}\right]=(.25) \frac{t^{2 \alpha}}{\sqrt{(1+2 \alpha)}}, \\
y^{(3)}=J_{t}^{\alpha}\left[y^{(0)} x^{(2)}-y^{(2)}+y^{(2)} x^{(0)}+y^{(1)} x^{(1)}\right]=(.125) \frac{t^{3 \alpha}}{\sqrt{(1+3 \alpha)}}
\end{gathered}
$$


hence the solution of $(16)$ is given by

$$
\begin{gathered}
x(t)=\sum_{i=0}^{\infty} x^{(i)}(t)=1+(0.5) \frac{t^{\alpha}}{\sqrt{(1+\alpha)}}+(0.25) \frac{t^{2 \alpha}}{\sqrt{(1+2 \alpha)}}+(.125) \frac{t^{2 \alpha}}{\sqrt{(1+2 \alpha)}}+\ldots \\
y(t)=\sum_{i=0}^{\infty} y^{(i)}(t) \quad=(0.5)+(0.25) \frac{t^{2 \alpha}}{\sqrt{(1+2 \alpha)}}+(.125) \frac{t^{2 \alpha}}{\sqrt{(1+2 \alpha)}}+\ldots
\end{gathered}
$$

This is the accurate and exact solution of the system (16), [7], using Ho motopy Perturbation Method.

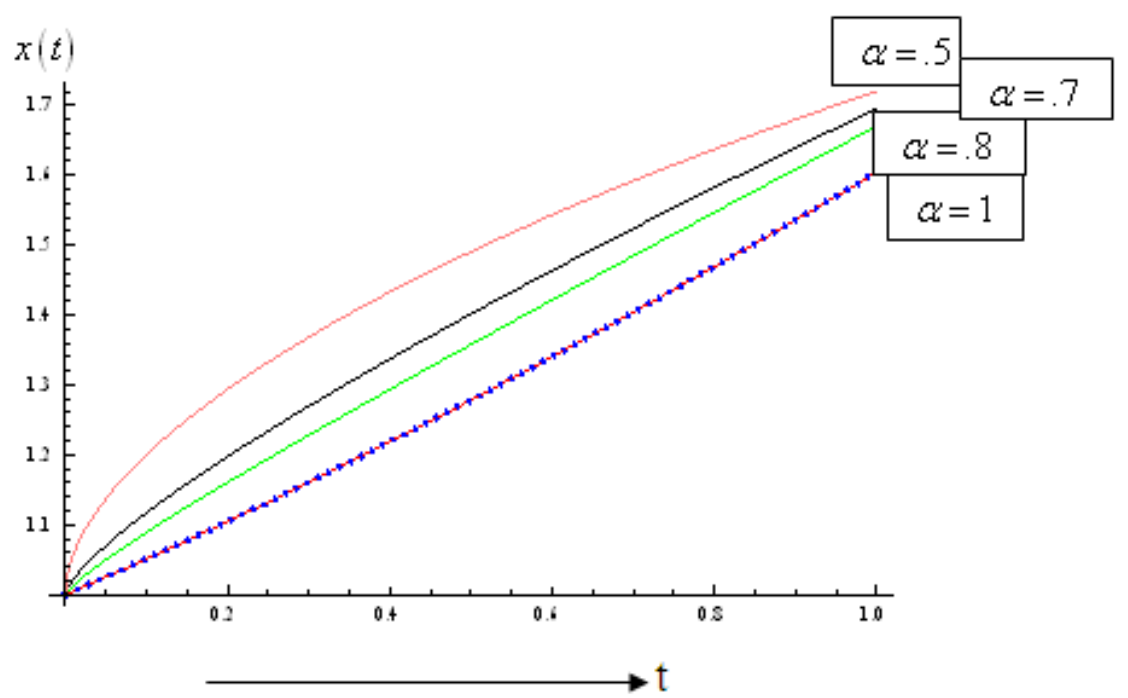

Figure 3. Plot of $x(t)$ vs $t$

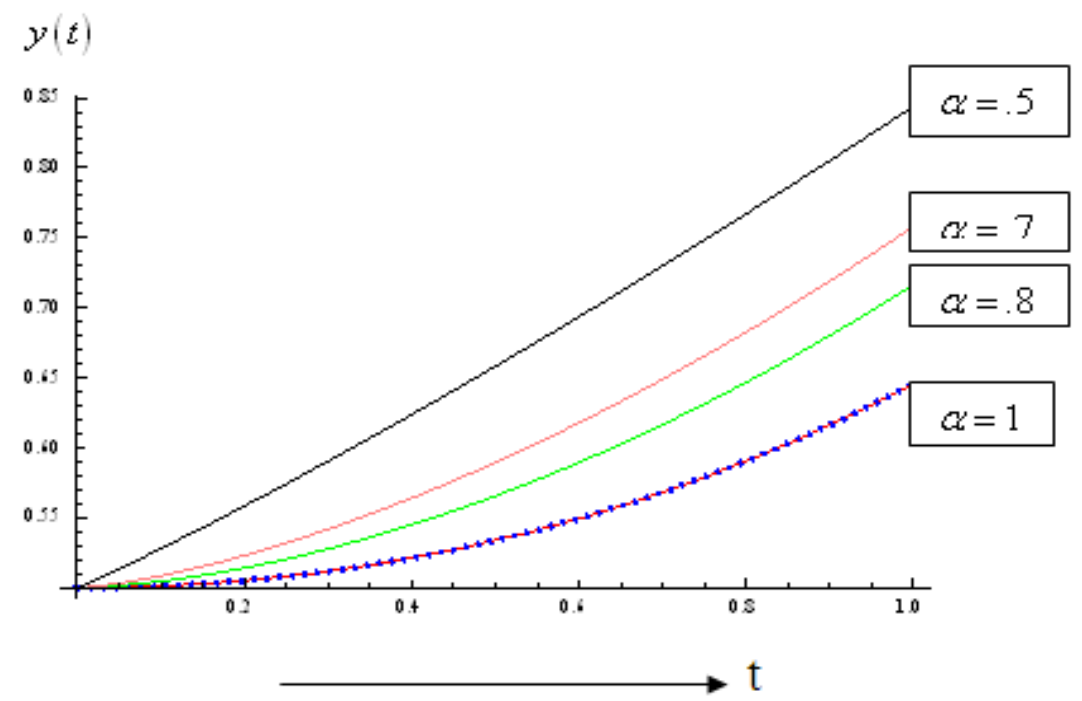

Figure 4. Plot of $y(t)$ vs $t$

From the Fig. 3 and Fig. 4 we see that the solution obtained by the proposed algorithm for $\alpha=1$ is same as the solution obtained by [16]. Also fig. 3 and 4 show the solution for other different values of $\alpha$.

Figure 3 and 4 show the solutions of HPM to the ecosystem model by red colour and that of the fractional ecosystem model by blue dots.

Example 4.3. Consider the predator - prey system 


$$
\begin{gathered}
D^{\alpha} x(t)=a x(t)-b x(t) y(t)-c x(t) z(t), \\
D^{\alpha} y(t)=-d y(t)+e x(t) y(t)-f y(t) z(t), \\
D^{\alpha} z(t)=-g z(t)+h x(t) z(t)-i y(t) z(t),
\end{gathered}
$$

where $a, b, c, d, e, f, g, h$ and $i$ are constants, subject to the initial conditions

$$
x(0)=c_{1}, y(0)=c_{2}, \quad z(0)=c_{3},
$$

We construct the Homotopy

$$
\begin{aligned}
& D^{\alpha} x(t)=p(a x(t)-b x(t) y(t)-c x(t) z(t)), \\
& D^{\alpha} y=p(-d y(t)+e x(t) y(t)-f y(t) z(t)), \\
& D^{\alpha} z(t)=p(-g z(t)+h x(t) z(t)-i y(t) z(t)),
\end{aligned}
$$

To solve the system given in Eq. (22) we construct the Homotopy as given in Eq. (7) putting Eq. (5) then intial condition (23) in Eq. (7) and then comparing the like powers of $p$ we get,

$$
\begin{aligned}
& p^{0}: D^{\alpha} x^{(0)}=0, p^{0}: D^{\alpha} y^{(0)}=0, p^{0}: D^{\alpha} z^{(0)}=0, \\
& p^{1}: D^{\alpha} x^{(1)}=a x^{(0)}-b x^{(0)} y^{(0)}-c x^{(0)} z^{(0)}, \\
& p^{1}: D^{\alpha} y^{(1)}=-d y^{(0)}+e x^{(0)} y^{(0)}-f y^{(0)} z^{(0)}, \\
& p^{1}: D^{\alpha} z^{(1)}=-g z^{(0)}+h x^{(0)} z^{(0)}-i y^{(0)} z^{(0)}, \\
& p^{2}: D^{\alpha} x^{(2)}=a x^{(1)}-b\left(x^{(0)} y^{(1)}+x^{(1)} y^{(0)}\right)-c\left(x^{(0)} z^{(1)}+x^{(1)} z^{(0)}\right), \\
& p^{2}: D^{\alpha} y^{(2)}=-d y^{(1)}+e\left(x^{(0)} y^{(1)}+x^{(1)} y^{(0)}\right)-f\left(y^{(0)} z^{(1)}+y^{(1)} z^{(0)}\right), \\
& p^{2}: D^{\alpha} z^{(2)}=-g z^{(1)}+h\left(x^{(0)} z^{(1)}+x^{(1)} z^{(0)}\right)+i\left(y^{(0)} z^{(1)}+y^{(1)} z^{(0)}\right),
\end{aligned}
$$

Using the operator $J_{t}^{\alpha}$ the inverse operator of $D^{\alpha}$ on both side of linear equations from $(24)-(26)$ with initial condition (23) we get,

$$
\begin{gathered}
x^{(0)}=x^{(0)}(0)=c_{1}, \\
x^{(1)}=J_{t}^{\alpha}\left[a x^{(0)}-b x^{(0)} y^{(0)}-c x^{(0)} z^{(0)}\right]=c_{1}\left(a-b c_{2}-c c_{3}\right) \frac{t^{\alpha}}{\sqrt{(1+\alpha)}} \\
x^{(2)}=J_{t}^{\alpha}\left[a x^{(1)}-b\left(x^{(0)} y^{(1)}+x^{(1)} y^{(0)}\right)-c\left(x^{(0)} z^{(1)}+x^{(1)} z^{(0)}\right)\right] \\
=\left(\begin{array}{l}
a c_{1}\left(a-b c_{2}-c c_{3}\right)-b c_{1} c_{2}\left(-d+e c_{1}-f c_{3}+a-b c_{2}-c c_{3}\right) \\
-c c_{1} c_{2}\left(-g+h c_{1}+i c_{2}+a-b c_{2}-c c_{3}\right)
\end{array}\right) \frac{t^{2 \alpha}}{\overline{(1+2 \alpha)}} \\
y^{(0)}=y^{(0)}(0)=c_{2}, \\
y^{(1)}=J_{t}^{\alpha}\left[-d y^{(0)}+e x^{(0)} y^{(0)}-f y^{(0)} z^{(0)}\right]=c_{2}\left(-d-e c_{1}-f c_{3}\right) \frac{t^{\alpha}}{\sqrt{(1+\alpha)}} \\
y^{(2)}=J_{t}^{\alpha}\left[-d y^{(1)}+e\left(x^{(0)} y^{(1)}+x^{(1)} y^{(0)}\right)-f\left(y^{(0)} z^{(1)}+y^{(1)} z^{(0)}\right)\right] \\
=\left(\begin{array}{l}
-d c_{2}\left(\left(-d+e c_{1}-f c_{3}\right)+e c_{1} c_{2}\left(-d+e c_{1}-f c_{3}+a-b c_{2}-c c_{3}\right)\right)- \\
f c_{2} c_{3}\left(-g+h c_{1}+i c_{2}-d+e c_{1}-f c_{3}\right)
\end{array}\right) \frac{t^{2 \alpha}}{\overline{(1+2 \alpha)}}
\end{gathered}
$$




$$
\begin{gathered}
z^{(0)}=z^{(0)}(0)=c_{3}, \\
z^{(1)}=J_{t}^{\alpha}\left[-g z^{(0)}+h x^{(0)} z^{(0)}-i y^{(0)} z^{(0)}\right]=c_{3}\left(-g+h c_{1}-i c_{2}\right) \frac{t^{\alpha}}{\sqrt{(1+\alpha)}}, \\
z^{(2)}=J_{t}^{\alpha}\left[-g z^{(1)}+h\left(x^{(0)} z^{(1)}+x^{(1)} z^{(0)}\right)+i\left(y^{(0)} z^{(1)}+y^{(1)} z^{(0)}\right)\right] \\
=\left(\begin{array}{l}
-g c_{3}\left(\left(-g+h c_{1}-i c_{2}\right)+h c_{1} c_{3}\left(-g+h c_{1}+i c_{2}+a-b c_{2}-c c_{3}\right)\right) \\
+i c_{2} c_{3}\left(-g-d+h c_{1}+i c_{2}+e c_{1}-f c_{3}\right)
\end{array}\right) \frac{t^{2 \alpha}}{\sqrt{(1+2 \alpha)}}
\end{gathered}
$$

Hence the solution is given as

$$
\begin{gathered}
x(t)=\sum_{i=0}^{\infty} x^{(i)}(t) \\
=c_{1}+c_{1}\left(a-b c_{2}-c c_{3}\right) \frac{t^{\alpha}}{\sqrt{(1+\alpha)}}+\left(\begin{array}{l}
a c_{1}\left(a-b c_{2}-c c_{3}\right)-b c_{1} c_{2}\left(-d+e c_{1}-f c_{3}+a-b c_{2}-c c_{3}\right) \\
-c c_{1} c_{2}\left(-g+h c_{1}+i c_{2}+a-b c_{2}-c c_{3}\right)
\end{array}\right) \frac{t^{2 \alpha}}{\sqrt{(1+2 \alpha)}} \\
y(t)=\sum_{i=0}^{\infty} y^{(i)}(t) \\
+c_{2}+c_{2}\left(-d-e c_{1}-f c_{3}\right) \frac{t^{\alpha}}{\sqrt{(1+\alpha)}} \\
+\left(\begin{array}{l}
-d c_{2}\left(\left(-d+e c_{1}-f c_{3}\right)+e c_{1} c_{2}\left(-d+e c_{1}-f c_{3}+a-b c_{2}-c c_{3}\right)\right) \\
-f c_{2} c_{3}\left(-g+h c_{1}+i c_{2}-d+e c_{1}-f c_{3}\right)
\end{array}\right) \frac{t^{2 \alpha}}{\sqrt{(1+2 \alpha)}} \\
=c_{3}+c_{3}\left(-g+h c_{1}-i c_{2}\right) \frac{t^{\alpha}}{\sqrt{(1+\alpha)}} \\
+\left(-g c_{3}\left(\left(-g+h c_{1}-i c_{2}\right)+h c_{1} c_{3}\left(-g+h c_{1}+i c_{2}+a-b c_{2}-c c_{3}\right)\right)+i c_{2} c_{3}\left(-g-d+h c_{1}+i c_{2}+e c_{1}-f c_{3}\right)\right) \frac{t^{2 \alpha}}{\sqrt{(1+2 \alpha)}}
\end{gathered}
$$

which is the accurate /exact solution of the system (22) using Homotopy Perturbation Method.

Example 4.4. Consider the system [17]

$$
\begin{gathered}
D^{\alpha} x(t)=a_{0} x(t)-b_{0} x^{2}(t)-\frac{v_{0} x(t) y(t)}{d_{0}+x(t)}, \\
D^{\alpha} y(t)=-a_{1} y(t)+\frac{v_{1} x(t) y(t)}{d_{1}+x(t)}-\frac{v_{2} y(t) z(t)}{d_{2}+y(t)}, \\
D^{\alpha} z(t)=a_{2} z(t)-\frac{v_{3} z^{2}(t)}{d_{3}+y(t)},
\end{gathered}
$$

Where $a_{0}, b_{0}, d_{0}, v_{0}, a_{1}, v_{1}, d_{1}, a_{2}, v_{2}, d_{2}, v_{3}$, and $d_{3}$ are model parameters assuming only positive values, with the in itial conditions

$$
x(0)=c_{1}, \quad y(0)=c_{2}, \quad z(0)=c_{3},
$$

multiplying the first equation of $(27)$ by the factor $\left(d_{0}+x(t)\right)$, the second equation of $(27)$ by the factor $\left(\left(d_{1}+x(t)\right),\left(d_{2}+y(t)\right)\right)$ and the third equation $(27)$ by the factor $\left(d_{3}+y(t)\right)$ To solve the systemgiven in Eq. 
(27) we construct the Homotopy as given in Eq. (7) putting Eq. (5) then intial condition (28) in Eq. (7) and then comparing the like powers of $p$ we get,

$$
\begin{gathered}
p^{0}: D^{\alpha} x^{(0)}=0, \quad p^{0}: D^{\alpha} y^{(0)}=0, \quad p^{0}: D^{\alpha} z^{(0)}=0, \\
p^{1}:\left(d_{0}+x^{(0)}\right) D^{\alpha} x^{(1)}=a_{0} d_{0} x^{(0)}+a_{0}\left(x^{(0)}\right)^{(2)}-b_{0} d_{0}\left(x^{(0)}\right)^{(2)}-b_{0}\left(x^{(0)}\right)^{(3)}-v_{0} x^{(0)} y^{(0)}, \\
p^{1}:\left(d_{1} d_{2}+d_{1} y^{(0)}+d_{2} x^{(0)}+x^{(0)} y^{(0)}\right) D^{\alpha} y^{(1)}= \\
-a_{1} d_{1} d_{2} y^{(0)}-a_{1} d_{1}\left(y^{(0)}\right)^{(2)}-a_{1} d_{2} x^{(0)} y^{(0)}-a_{1} x^{(0)}\left(y^{(0)}\right)^{(2)} \\
+v_{1} d_{2} x^{(0)} y^{(0)}+v_{1} x^{(0)}\left(y^{(0)}\right)^{(2)}-v_{2} d_{1} y^{(0)} z^{(0)}-v_{2} x^{(0)} y^{(0)} z^{(0)} \\
p^{1}:\left(d_{3}+y^{(0)}\right) D^{\alpha} z^{(1)}=-a_{2} d_{3} z^{(0)}+a_{2} y^{(0)} z^{(0)}-v_{3}\left(z^{(0)}\right)^{(2)}
\end{gathered}
$$

using the operator $J_{t}^{\alpha}$ the inverse operator of $D^{\alpha}$ on both side of linear equations from $(29)-(30)$ with initial condition (28) we obtained,

$$
\begin{aligned}
& x^{(0)}=x^{(0)}(0)=c_{1}, \\
& x^{(1)}=J_{t}^{\alpha}\left[\frac{a_{0} d_{0} x^{(0)}+a_{0}\left(x^{(0)}\right)^{(2)}-b_{0} d_{0}\left(x^{(0)}\right)^{(2)}-b_{0}\left(x^{(0)}\right)^{(3)}-v_{0} x^{(0)} y^{(0)}}{\left(d_{0}+x^{(0)}\right)}\right] \\
& =\left(a_{0} c_{1}-b_{0}\left(c_{1}\right)^{(2)}-\frac{v_{0} c_{1} c_{3}}{d_{0}+c_{1}}\right) \frac{t^{\alpha}}{\overline{(1+\alpha)}} \\
& y^{(0)}=y^{(0)}(0)=c_{2}, \\
& y^{(1)}=J_{t}^{\alpha}\left[\frac{-v_{2} d_{1} y^{(0)} z^{(0)}-v_{2} x^{(0)} y^{(0)} z^{(0)}}{\left(d_{1} d_{2}+d_{1} y^{(0)}+d_{2} x^{(0)}+x^{(0)} y^{(0)}\right)}\right] \\
& =\left(-a_{1} c_{2}+\frac{v_{1} c_{1} c_{2}}{d_{1}+c_{1}}-\frac{v_{2} c_{2} c_{3}}{d_{2}+c_{2}}\right) \frac{t^{\alpha}}{\sqrt{(1+\alpha)}} \\
& z^{(0)}=z^{(0)}(0)=c_{3}, \\
& z^{(1)}=J_{t}^{\alpha}\left[\frac{-a_{2} d_{3} z^{(0)}+a_{2} y^{(0)} z^{(0)}-v_{3}\left(z^{(0)}\right)^{(2)}}{\left(d_{3}+y^{(0)}\right)}\right] \\
& =\left(a_{2} c_{3}-\frac{v_{3}\left(c_{3}\right)^{(2)}}{d_{3}+c_{2}}\right) \frac{t^{\alpha}}{\sqrt{(1+\alpha)}}
\end{aligned}
$$

Hence the solution is given by 


$$
\begin{gathered}
x(t)=\sum_{i=0}^{\infty} x^{(i)}(t)=c_{1}+\left(a_{0} c_{1}-b_{0}\left(c_{1}\right)^{(2)}-\frac{v_{0} c_{1} c_{3}}{d_{0}+c_{1}}\right) \frac{t^{\alpha}}{\sqrt{(1+\alpha)}}+\ldots \\
y(t)=\sum_{i=0}^{\infty} y^{(i)}(t)=c_{2}+\left(-a_{1} c_{2}+\frac{v_{1} c_{1} c_{2}}{d_{1}+c_{1}}-\frac{v_{2} c_{2} c_{3}}{d_{2}+c_{2}}\right) \frac{t^{\alpha}}{\sqrt{(1+\alpha)}}+\ldots \\
z(t)=\sum_{i=0}^{\infty} z^{(i)}(t)=c_{3}+\left(a_{2} c_{3}-\frac{v_{3}\left(c_{3}\right)^{(2)}}{d_{3}+c_{2}}\right) \frac{t^{\alpha}}{\sqrt{(1+\alpha)}}+\ldots
\end{gathered}
$$

This is closed form of solution of the system (27) using HPM.

\section{Conclusions}

In this paper we have introduced an algorithm based Homotopy Perturbation Method to solve the fractional ecosystem model. The method has been applied to get exact and accurate solution for linear and nonlinear systems of fractional Ecosystem model, the solution obtained matches with the solution of the orig inal ecosystem for $\alpha=1$ and both the variables (populations) increases with time while value of $\alpha$ decreases and the solution converges to the original solution of the linear and nonlinear ecosystem model as the fractional parameter $\alpha$ tends to 1 as found in two examples. This method can also be applied to similar linear and nonlinear models in other systems. Further it is also verified that the FHPM is more efficient than HPM.

\section{ACKNOWLEDGEMENTS}

The first author expresses thanks to fellow scholars, in particular to Rajneesh Kumar, Vipul Ku mar Barn wal and Dr. $\mathrm{S}$ Das who helped him in preparation of the present paper.

\section{REFERENCES}

[1] J. B. Waide, and J. R. Webster, "En gineering systems analy sis: Applicability to ecosystems", Systems analysis and simulation in ecology. Vol.4., pp. 329-371., Acad. Press, N.Y, 1976.

[2] J. A. Wiens, and G. S. Innis. "Estimation of energy flow in bird communities." , A population bioenergetics model Ecology , 55:730-746. Marine Fisherie, 1974.

[3] K. A. Rose, "A review and comparison of parameter sensitivity methods applicable to large simulation models",50 p .M.S. Thesis Univ. Wash., Seattle, 1981.

[4] "Its quantitative evaluation and management", Marine fisheries ecosystem Fishing 162 p. News Books Ltd., Farnham, Surrey, England.
[5] R. H.Gardner, R. V. O Neill, J. B. Mankin,and O. Kumar. "Comparative error analysis of six predator-prey models", Ecology. $61: 323-332.1980$.

[6] S. Momani, Z. Odibat, "Numerical comparison of methods for solving linear differential equations of fractional order",Chaos, Soli tons and Fractals, doi:10.1016/j. chaos.10.068. 2005.

[7] S.S. Ray, R.K. Bera, “An approximate solution of a nonlinear fractional differential equation by Adomian decomposition method", Appl. Math. Comput. 167, 561-571, 2005.

[8] N.T. Shawagfeh, "Analytical approximate solutions for nonlinear fractional differential equations", Appl. Math. Comput.131, 517-529, 2002.

[9] I. Podlubny, "Fractional Differential Equations", Academic Press, NewYork, 1999.

[10] R.Gorenflo, F.Mainardi, "Fractional Calculus Integral and Differential Equations of Fractional Order", Springer, Wien and NewYork, 1997.

[11] S. Abbasbandy, "Homotopy perturbation method for quadratic Riccati differential equation and comparison with Adomian's decomposition method", Appl. Math. Comp., 172, 485-490, 2006.

[12] Z. Odibat and S. Momani "Modified homotopy pertur-bation method application to quadratic Riccati differential equation of fractional order" Chaos Solitons \& Fractals, (in press).

[13] J. He "Homtopy perturbation method a new non-linear analy tic technique" Appl. Math. Comp. 135, 73-79, 2003.

[14] J. He "Application of homotopy perturbation method to nonlinear wave equations" Chaos, Solitons \& Fractals, 26(3), 695-700, 2005.

[15] O. Abdulaziza, I. Hashima,, S. Momani, "Application of homotopy-perturbation method to fractional IVPs", J.Computational and Applied Mathematics, 216,574-584, 2008.

[16] S. Momani and Z Odibat. "Numerical approch to differential equations of fractional order" J. Comput. Appl.Math. (in press).

[17] M.A. Aziz-Alaoui "Complex emergent properties and choas (de-) synchronization". in M.A. Aziz-Alaoui and C. Bertelle (eds), "Emergent Properties in Natural and Artificial Dynamical Systems", Springer, 2006. 